\title{
In vivo immunofluorescence to diagnose herpes simplex virus keratitis in mice
}

\author{
A Sharma, C Shimeld
}

\begin{abstract}
Backgroundlaims-Herpes simplex virus keratitis (HSK) is the most common cause of corneal blindness in the Western world. Delay in the treatment of HSK can lead to a more significant corneal scar and topical steroid treatment in unsuspected active HSK can lead to corneal melting. Current culture techniques for herpes simplex virus (HSV) take several days and commercially available HSV laboratory based diagnostic techniques such as Herpchek vary in sensitivity. This study was conducted to assess the viability of a new, quicker, and simpler method to diagnose HSK.

Methods-Direct immunofluorescence was used in vivo in a masked study to diagnose HSK in mice using a standard slit lamp with cobalt blue illumination. Murine monoclonal fluorescently labelled antibody was applied to the cornea for $\mathbf{1 0}$ or 20 minutes and then washed off with phosphate buffered solution. Mice with HSK were stained with either fluorescently labelled monoclonal antibody against HSV or fluorescently labelled monoclonal antibody against cytomegalovirus. Mice with corneal abrasions of nonviral origin were given fluorescently labelled monoclonal antibody against HSV.

Results-Fluorescence was seen only in the mice with HSK given fluorescently labelled monoclonal antibody against HSV. This observation was confirmed upon microscopic immunofluorescent imaging of the corneal epithelial sheets. Conclusion-In vivo immunofluorescence may be useful in the clinical diagnosis of HSK.
\end{abstract}

Medical Microbiology, University of Wales college of Medicine, Cardiff CF4 4XW

A Sharma

Department of Ophthalmology, Medical Sciences Building, University Walk, Bristol BS8 1TD C Shimeld

Correspondence to: Mr A Sharma, Cardiff Eye Unit, University Hospital of Wales, Cardiff, South Glamorgan CF4 4XW.

Accepted for publication 28 May 1997

most common cause of corneal blindness in the Western world. ${ }^{1}$ Approximately $11 \%$ of corneal grafts carried out in the UK and the Republic of Ireland are undertaken because of HSK. ${ }^{2}$ Correct diagnosis of keratitis is important for appropriate patient management. Topical steroid treatment is used to prevent corneal graft rejection and for the treatment of corneal diseases such as metaherpetic ulcers and marginal keratitis. In such clinical situations active herpes simplex virus (HSV) infection may be suspected and steroids may be withheld or reduced. Topical steroids exacer- bate active HSV infection and can lead to corneal perforation.

Conversely, other conditions such as healing epithelial defects, Acanthamoeba keratitis, and soft contact lens wear keratopathy may mimic $\mathrm{HSK}^{3-6}$ and antiviral agents may be started.

Current culture techniques for HSV take several days and commercially available HSV diagnostic techniques such as Herpchek (Dupont, Wilmington, DE, USA) vary in sensitivity from $65 \%{ }^{7}$ to $100 \%{ }^{8}$ Improved Herpchek is a 5 hour test, and is not suitable for routine use in an office practice but requires a central laboratory. ${ }^{8}$

Immunofluorescence staining techniques are used routinely in vitro to diagnose infections especially viruses including HSV. The mouse model has been well established for studying HSK, HSV type 1 (HSV-1) shedding in the tear film, and HSV-1 antigen in the corneal epithelium. ${ }^{910}$ The inoculation method used in this study has been shown to produce HSK in all mice: on day 3 after inoculation all mice have dendritic ulcers and eye washings from all mice were positive for HSV- $1 .{ }^{11}$ The following study was conducted to assess the viability of in vivo direct immunofluorescence as a diagnostic technique for HSK.

\section{Materials and methods}

ANIMALS

Female, 8-week-old, albino, inbred NIH mice were obtained from Harlan/Olac (Bicester, Oxford). Before inoculation both eyes were examined by a slit lamp (Zeiss USL) and any with abnormal eyes were rejected. After inoculation the mice were changed from sawdust to shredded newspaper bedding to avoid risk of ocular damage.

\section{ANAESTHESIA}

Midazolam (Hypnovel) (Roche) and fentanyl citrate/fluanisone (Hypnorm) from Chemicals Co Ltd were administered in the scruff of the neck, as two separate subcutaneous $0.1 \mathrm{ml}$ injections (midazolam hydrochloride $2.4 \mathrm{mg}$ / $\mathrm{kg}$, fluanisone $4.8 \mathrm{mg} / \mathrm{kg}$, and fentanyl citrate $0.15 \mathrm{mg} / \mathrm{kg}$ ).
INOCULATION

Mice were anaesthetised and while viewing through a binocular microscope (Vickers) they were inoculated by scarification of the left cornea through a $5 \mu$ drop of maintenance medium containing HSV type 1 (HSV-1) strain McKrae. The dose was $1 \times 10^{4}$ plaque forming units (pfu). The scarification was done immediately after the inoculum was instilled. 
Five linear corneal scarifications and a further five perpendicular to the first, were made with a 26 gauge $\times 3 / 8$ inch needle (Becton Dickinson).

On the day of examination, lesions were made in the corneal epithelium of control mice with a 26 gauge needle.

ANTIBODIES

Three different murine monoclonal antibodies $(\mathrm{MoAb})$ labelled with fluorescein isothiocyanate (MoAb-F) class IgG1 were used (obtained from Biogenesis Ltd, Poole); these antibodies were dialysed to remove preservative and stored at $4^{\circ} \mathrm{C}$. The fluorescein isothiocyanate (FITC) bound to antibodies has the same absorption peak (approximately $495 \mathrm{~nm}$ ) as fluorescein used clinically to stain corneal epithelial defects. The first MoAb was directed against HSV-1 envelope glycoprotein $\mathrm{gB}$, the second $\mathrm{MoAb}$ recognised a cytoplasmic protein in HSV-1 infected cells, and the third $\mathrm{MoAb}$ was directed against cytomegalovirus (CMV). The MoAbs were labelled with FITC to a molar fluorescein to IgG ratio of 8:1 for the $\mathrm{MoAb}$ against $\mathrm{HSV}-1$ envelope glycoprotein $\mathrm{gB}, 2: 1$ for the $\mathrm{MoAb}$ against a cytoplasmic protein in HSV-1 infected cells, and 5:1 for the MoAb against CMV. A combination of the $\mathrm{MoAb}$ against HSV-1 envelope glycoprotein $\mathrm{gB}$ and $\mathrm{MoAb}$ against a cytoplasmic protein in HSV-1 infected cells was used at a concentration of $2 \mathrm{mg} / \mathrm{ml}$. These two combined MoAb-Fs will be referred to as combined HSV-1 MoAb-Fs. The concentration of the $\mathrm{MoAb}$ against CMV (CMV MoAb-F) was $0.67 \mathrm{mg} / \mathrm{ml}$.

To assess the specificity of the MoAbs used, human fibroblast monolayers were infected with HSV-1 strain McKrae. At approximately $30 \%$ to $50 \%$ cytopathic effect the cells were resuspended and slides made and stained with the MoAb-Fs. Both of the above MoAb-Fs, comprising the combined HSV-1 MoAb-Fs, showed very good staining of virus infected cells at a dilution of 1:20 in vitro. The CMV $\mathrm{MoAb}-\mathrm{F}$ did not show staining of virus infected cells at this dilution.

IN VIVO IMMUNOFLUORESCENCE STAINING

Three or 4 days after inoculation of virus, mice were anaesthetised and $5 \mu \mathrm{l}$ of MoAb-F placed onto the left cornea; after 10 or 20 minutes the antibody was washed off with phosphate buffer solution (PBS). The eyes were examined with a Nikon FS-3 slit lamp in white and cobalt blue light. The magnification used was up to 32.3 times normal. Agfa 1000 ISO colour film was used.

The eyes that showed fluorescence on the slit lamp were re-examined half an hour later to assess the duration of fluorescence.

The mice were killed and the eyes were enucleated and incubated in EDTA 2\% for 2 hours at $37^{\circ} \mathrm{C}$ to aid removal of the epithelium, and then transferred to PBS. While viewing with a dissecting microscope the epithelium was removed as a sheet from the eye using fine forceps. ${ }^{9}$ The epithelial sheets were mounted on glass slides and dried and viewed with a fluorescent microscope (Zeiss photomicroscope 2 with a 3 RS epifluorescence attachment and a HBO 50 WAC lamp). The observer was masked as to which mice had been inoculated with HSV-1 and which antibodies had been applied to the mice.

Sixteen eyes of 14 mice were examined in total. Ten eyes were inoculated with HSV-1, four had uninfected corneal abrasions, and two were normal corneas. Six mice had HSK and combined HSV-1 MoAb-Fs placed onto the left cornea, three mice for 10 minutes and the others for 20 minutes; four mice had HSK and CMV MoAb-F placed on their eye, two mice for 10 minutes and the others for 20 minutes; four mice had corneal abrasions with combined HSV-1 MoAb-Fs placed on their eye, two mice for 10 minutes and the others for 20 minutes. The normal (right) corneas of two of the above mice had combined HSV-1 MoAb-Fs placed on them, one mouse for 10 minutes and the other for 20 minutes. Mice were placed in a dark cage (at room temperature) during the incubation time with the antibody. The HSK and abrasions were produced by, and the MoAb-Fs placed on the eye by, a different observer from the one examining the eyes with the slit lamp.

\section{Results}

All the HSV-1 infected corneas had significant ulceration at the time of application of the MoAb-Fs.

There was no evidence of fluorescence, when the corneas were viewed with the slit lamp, in the following control mice; mice with corneal abrasions or mice with normal corneas which had combined HSV-1 MoAb-Fs applied or animals with HSK that had CMV MoAb-F applied.

Three of the six eyes with HSK and combined HSV-1 MoAb-Fs showed areas of fluorescence seen with the slit lamp at 32.3 times magnification. In all of these three eyes in which fluorescence was seen with the slit lamp the fluorescence was associated with disease; in one of the three cases (mouse 1, Fig 1) the fluorescence was bifocal at the margin of the HSK ulcer and in the other two mice (mice 3 and 11) the fluorescence was multifocal at the margins of the ulcers and granular in appearance. Figure 1 shows the focal nature of fluorescence that was seen in mouse 1. The focal fluorescence in all of these three mice (mice 1, 3, and 11) remained when reviewed half an hour later with the slit lamp using cobalt blue light.

On fluorescent microscopy of the corneal epithelial sheets only non-specific diffuse fluorescence (Fig 2) or no fluorescence was seen in the following control mice; mice with corneal abrasions or mice with normal corneas that had combined HSV-1 MoAb-Fs applied or animals with HSK that had CMV MoAb-F applied.

Specific microscopic fluorescence was seen in the epithelial sheets from three of six mice with HSK treated with combined HSV-1 MoAb-Fs (mice 1, 3, and 11). These were the same mice that showed positive fluorescence in 


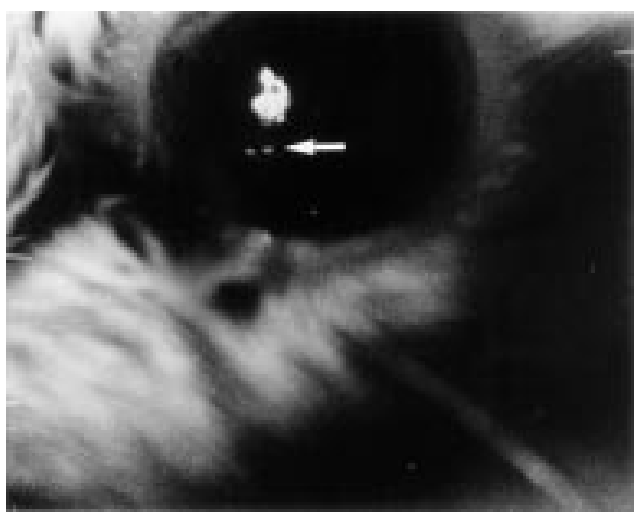

Figure 1 Mouse 1. Mouse eye treated with combined $H S V-1 M o A b-F s, 3$ days after inoculation of the cornea with HSV-1. Bifocal nature of immunofluorescence (arrow) seen below the light reflex. Slit lamp magnification 32.3 times with cobalt blue illumination.

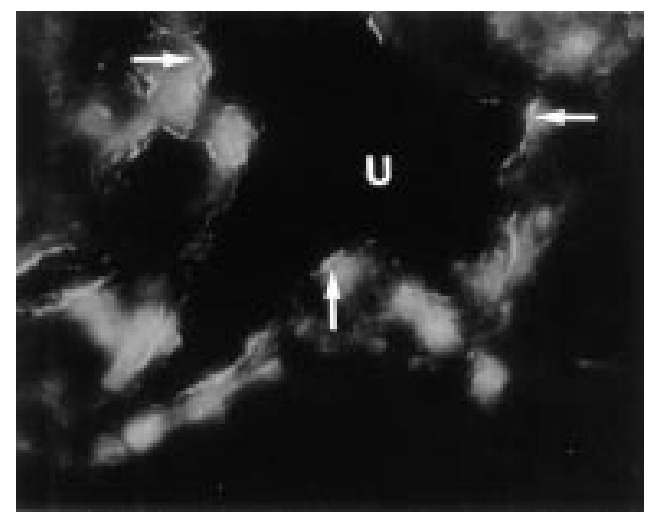

Figure 3 Mouse 3. The corneal epithelial sheet from a mouse eye treated with combined HSV-1 MoAb-Fs, 3 days after inoculation of the cornea with HSV-1. Microscopic immunofluorescence $(\times 42)$. Specific linear fluorescence (arrows) outlining the ulcer (U).

vivo. Mice 1 and 3 showed linear fluorescence outlining an ulcer (Figs 3 and 4). Mouse 11 showed focal fluorescence.

The durations of application of antibody in the three mice that showed fluorescence in vivo and specific microscopic fluorescence of their corneal epithelial sheets were, in mice 1 and 11,10 minutes, and in mouse 3, 20 minutes.

\section{Discussion}

HSV-1 replicates in host cells and expresses envelope glycoprotein in the infected host cell membrane. We assumed that a MoAb-F against a HSV-1 envelope glycoprotein would be an important MoAb-F in vivo to detect HSV-1 infection, as it would bind to the corresponding HSV-1 envelope glycoprotein found in infected cell surface membranes. MoAb-F against HSV-1 antigen present in the cytoplasm of infected cells was also used to attempt to increase the number of virus infected cells detected, assuming some infected cells would be damaged and allow antibody to penetrate. Our study used a higher fluorescein to antibody ratio than is conventionally used for in vitro staining. This may have resulted in non-specific staining; but this was not the case in this study.

These results show that the in vivo immunofluorescent technique correctly detected HSK

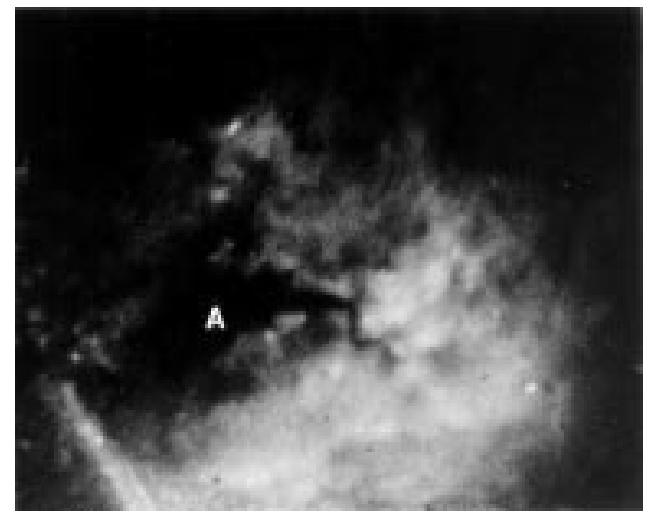

Figure 2 Mouse 6. The corneal epithelial sheet from a mouse eye with corneal abrasion treated with combined HSV-1 MoAb-Fs. Microscopic immunofluorescence (× 42). Non-specific diffuse fluorescence seen around a small abrasion $(A)$.

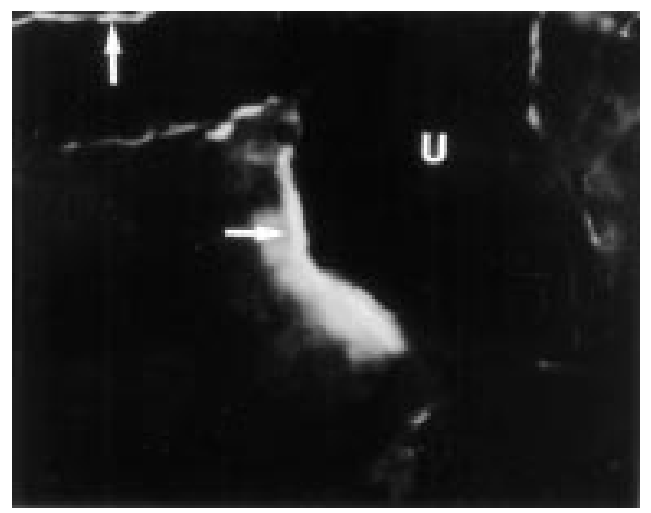

Figure 4 Mouse 3. The corneal epithelial sheet from a mouse eye treated with combined $H S V-1 M o A b-F s$, 3 days after inoculation of the cornea with HSV-1. Microscopic immunofluorescence $(\times 42)$. Specific linear fluorescence (arrows) outlining the ulcer (U).

in three out of six cases, giving a sensitivity of $50 \%$, though the number of eyes examined was too small to evaluate the true sensitivity of this test. The specificity was $100 \%(n=10)$ as none of our controls - mice with corneal abrasions or mice with normal corneas which had combined HSV-1 MoAb-Fs applied or animals with HSK that had CMV MoAb-F appliedshowed either fluorescence when viewed with the slit lamp or specific fluorescence of their corneal epithelial sheets under direct fluorescent microscopy. Again, the number of eyes examined was too small to evaluate the true specificity of this test. The sensitivity of our technique may be a result of different rates of virus antigen expression with time after inoculation. However, it has been shown that up to 3 days after infection the virus antigen in the corneal epithelium is maximum. ${ }^{9}$ The specific fluorescence, seen with the slit lamp and with microscopy, appears to be at the margins of the HSK ulcer where the HSV-1 antigen is expressed. ${ }^{9}$ Enhancement or improved observation of the fluorescein signal from the bound MoAb-F could improve the sensitivity of this technique. The fluorescein signal could be increased by maximising the binding of antibody to the HSV-1 infected cells by the use of a pool of MoAb-Fs targeted to different HSV-1 antigens or by using fluorescein labelled 
polyclonal antibody. Increased concentration of the MoAb-Fs and a higher fluorescein to antibody ratio than that used in this study could also improve the fluorescein signal. Although the indirect fluorescence method may improve the fluorescein signal we felt that this would be less applicable in the clinical situation and may also reduce the specificity by non-specific binding of the secondary fluorescein labelled antibody. Observation of the fluorescein signal could be improved by using higher magnification than that used in this study, or confocal microscopy.

In vivo immunofluorescence has been used to detect toxoplasmic chorioretinitis in rabbits 2 to 4 hours after the intravenous injection of 20-30 $\mathrm{mg}$ of anti-toxoplasma fluorescently labelled IgG antibodies. ${ }^{12}$ Our study used up to $10 \mu \mathrm{g}$ of MoAb-Fs applied topically in a masked study and the specific fluorescence was visible 10 or 20 minutes after application of the antibody. The ocular surface appears to be an ideal place to study disease with in vivo immunofluorescence, as a high concentration of antibody can be delivered topically to the area of interest with less dilution as would occur with intravenous application of fluorescently labelled antibody, and it can be viewed readily. Furthermore, foreign fluorescently labelled antibodies applied topically should induce far less of an immune response in the host than intravenous foreign antibodies. The authors feel that in vivo immunofluorescence may have a role to play in the clinical setting to diagnose ocular surface disease such as HSK, but further work needs to be done to investigate the host immune response to topical foreign antibodies.

1 Polack FM. In: External diseases of the eye. Barcelona: Ediciones Scriba, 1991:139.

2 Vail A, Gore SM, Bradley BA, Easty DL, Rogers CA. Corneal transplantation in the United Kingdom and Republic of Ireland. Br f Ophthalmol 1993;77:650-6.

3 Arffa RC. In: Grayson's diseases of the cornea. 3rd ed. St Louis: Mosby-Yearbook, 1991;215:326-8.

4 Margulies LJ, Mannis MJ. Dendritic corneal lesions associated with soft contact lens wear. Arch Ophthalmol 1983;101:1551-3.

5 Jones BR. Thygeson's superficial punctate keratitis. Trans Ophthalmol Soc UK 1963;83:245-53.

6 Charlton KH, Binder PS, Wozniak L, Digby DJ. Pseudodendritic keratitis and systemic tyrosinemia. Ophthalmology 1981;88:355-60.

7 Kowalski RP, Gordon YJ. Evaluation of immunological techniques for the detection of ocular herpes simplex virus. techniques for the detection of ocul

Ophthalmology 1989;96:1583-6.
8 Kowalski RP, Gordon YJ, Romanowski MS, Araullo-Cruz $\mathrm{T}$, Kinchington PR. A comparison of enzyme immunoassay and polymerase chain reaction with the clinical examination for diagnosing ocular herpetic disease. Ophthalmology 1993;100:530-3.

9 Shimeld C, Stanislawa J, Lewkowicz-Moss SJ, Lipworth KM, Hill TJ, Blyth WA, et al. Antigens of herpes simplex virus in whole corneal epithelial sheets from mice. Arch Ophthalmology 1986;104:1830-4

10 Tullo AB, Shimeld C, Blyth WA, Hill TJ, Easty DL. Ocular infection with herpes simplex virus in nonimmune and immune mice. Arch Ophthalmol 1983;101:961-4.

11 Shimeld C, Hill TJ, Blyth WA, Easty DL. Passive immunization protects the mouse eye from damage after herpes simplex infection by limiting spread of virus in the herpes simplex infection by limiting spread

12 Scheiffarth OF, Zrenner E, Disko R, Stefani FH, Brabander B. Intraocular in vivo immunofluorescence. Invest Ophthalmol Vis Sci 1990;31:272-6. 